\title{
A phase 1 evaluation of the pharmacokinetic/pharmacodynamic interaction of the anti-malarial agents KAF156 and piperaquine
}

\author{
F. Joel Leong ${ }^{1,5^{*}}$, Jay Prakash Jain ${ }^{2 *}$, Yiyan Feng ${ }^{3}$, Budhaditya Goswami ${ }^{2}$ and Daniel S. Stein ${ }^{4}$
}

\begin{abstract}
Background: KAF156 is a novel imidazolopiperazine anti-malarial with activity against pre-erythrocytic liver stages, asexual and sexual blood stages. Based on in vitro data, a two-way pharmacokinetic interaction was hypothesized for KAF156 use in combination with piperaquine (PPQ) as both drugs are CYP3A4 substrates and inhibitors. Potential combination effects on the QT interval were also assessed.

Methods: This was an open-label, parallel-group, single-dose study in healthy volunteers randomized to three parallel arms (1:1:1) of 800 mg KAF156 + 1280 mg PPQ, 800 mg KAF156 alone and 1280 mg PPQ alone. Triplicate ECGs were done up to $48 \mathrm{~h}$ post-dose. Routine safety and pharmacokinetic assessments were carried out up to 61 days.

Results: Of the 72 healthy male subjects recruited, 68 completed the study. Co-administration of PPQ and KAF156 had no overall effect on AUC of either compound, but the Cmax values of both KAF156 ( 23\%) and piperaquine $(\sim 70 \%)$ increased. Both drugs given alone or in combination were well tolerated with no deaths or serious adverse events (SAEs). AEs were observed at the frequency of 87.5, 79.2 and 58.3\% respectively for KAF156 + PPQ, PPQ and KAF156 arms. The most common AEs were nausea and headache. There were no Grade 3 or 4 events. There were no ECG related AEs, no QTcF interval > $480 \mathrm{~ms}$ and no QTcF interval increase from baseline $>60 \mathrm{~ms}$. There was a positive $\triangle \mathrm{QTCF}$ trend in the KAF156 + PPQ arm when either KAF156 or piperaquine concentration increases, but there was no significant difference between the combination arm and other arms in maximum $\triangle \mathrm{QTCF}$.
\end{abstract}

Conclusions: No safety/cardiac risk or drug interaction was identified which would preclude use of a KAF156 and PPQ combination in future studies.

Keywords: Malaria, QT interval, Piperaquine, KAF156

\section{Background}

Despite a significant decrease in incidence over the last 5 years, malaria continues to have a significant health impact with 212 million cases worldwide in 2015, mostly in sub-Saharan Africa in children less than 5 years of age. In 2016, 91 countries were endemic for malaria, with approximately half within the World Health Organization

\footnotetext{
*Correspondence: joel_leong@d3.a-star.edu.sg; jay_prakash.jain@ novartis.com

${ }^{1}$ Novartis Institute for Tropical Diseases, Singapore, Singapore

${ }^{2}$ Novartis Healthcare Pvt Ltd, Hyderabad, India

Full list of author information is available at the end of the article
}

(WHO) African region-sub-Saharan Africa experienced $90 \%$ of malaria cases and $92 \%$ of malaria deaths [1].

WHO guidelines recommend fixed dose combination therapy to decrease potential for development of resistance that can occur from either non-compliance or monotherapy. Drug resistance has occurred with all prior therapies developed for malaria and it has been detected to a limited extent for the artemisinin class, the current mainstay of treatment [2-7]. Despite emphasis on the use of combination therapies it is predicted that given the current incidence detection of artemisinin resistance in clinical settings [2], widespread artemisinin drug 
resistance will occur, as has been the historical experience for prior anti-malarials [8]. Therefore, development of new non-artemisinin based anti-malarial drugs is urgently needed.

KAF156 belongs to a novel class of anti-malarials (imidazolopiperazines) $[9,10]$. Through a mechanism that remains to be determined, it kills both blood and liver schizonts, has both therapeutic and causal prophylactic activities in malaria mouse models, and blocks transmission to the Anopheles mosquito. It demonstrates nanomolar half maximal inhibitory concentration (IC50) values against all lab-adapted Plasmodium falciparum drug resistant strains as well as Plasmodium vivax, and $P$. falciparum clinical isolates (IC50 range $=5-15 \mathrm{nM}$ ) [11]. It has been dosed in healthy adult volunteers up to $600 \mathrm{mg}$ daily for 3 days and $1200 \mathrm{mg}$ as a single dose [12]. It has also demonstrated efficacy in uncomplicated adult malaria patients. With a multiple-dose regimen $(400 \mathrm{mg}$ daily for 3 days) median parasite clearance times (PCT) were $45 \mathrm{~h}$ (interquartile range, $42-48$ ) in 10 patients with falciparum malaria and $24 \mathrm{~h}$ (interquartile range, 20-30) in 10 patients with vivax malaria. PCT was $49 \mathrm{~h}$ (interquartile range, $42-54$ ) in 21 falciparum malaria patients after treatment with a single $800 \mathrm{mg}$ dose [13].

KAF156 is absorbed rapidly with a Tmax of $1-4 \mathrm{~h}$. It has over-proportional exposure in the dose range of 10-1200 mg. There is no significant impact of food on KAF156 exposure. Its mean elimination half-life is in range of 47.1-55.6 h. Pharmacokinetic properties are similar in both malaria patients and healthy subjects $[12$, 13].

Piperaquine (PPQ) is an approved anti-malarial drug as part of the combination product Eurartesim ${ }^{\circledR}$ [14-17]. Piperaquine has long acting anti-malarial activity with relatively low levels of resistance, however, its major safety issue is a significant drug exposure-related increase in QT interval observed in trials that further increases when drug exposure is increased by food intake $[18,19]$.

KAF156 and piperaquine are both CYP3A4 inhibitors and primarily metabolized by CYP3A4. The inhibitory constant $(\mathrm{Ki})$ values determined from the in vitro CYP3A4 inhibition study were 0.156-0.09 $\mu \mathrm{M}$ for KAF156 and piperaquine, respectively (Novartis data on file). However, SimCYP ${ }^{\circledR}$ (Certara) modelling and simulation predicted no significant pharmacokinetic interaction for both KAF156 and piperaquine for in vivo combination use (i.e., < 1.25-fold change in AUC).

To evaluate piperaquine as a potential combination partner of KAF156 this study assessed the potential bidirectional interaction of KAF156 and piperaquine on each other's pharmacokinetics in healthy subjects, and assessed the safety, including the cardiac safety (QTc prolongation), when KAF156 and piperaquine were given alone or in combination.

\section{Methods \\ Study design}

This was an open label, randomized, single dose, parallelgroup and non-confirmatory study in healthy volunteers. It was conducted at Nucleus Network in Melbourne, Australia. The primary objective was to investigate the pharmacokinetic interaction potential between KAF156 and piperaquine in healthy subjects. Secondary objectives were to investigate the safety and tolerability of KAF156 and piperaquine alone and when co-administered in healthy subjects, and to investigate the potential effects on electrocardiogram (ECG) intervals (QT, PR, QRS) when KAF156 and piperaquine were given alone and in combination.

\section{Treatments and follow-up schedule}

The proposed doses were $800-1280 \mathrm{mg}$ for KAF156 and piperaquine, respectively. The KAF156 dose of $800 \mathrm{mg}$ (KAF156 base equivalent) was given in the form of eight $100 \mathrm{mg}$ strength tablets. Piperaquine was administered as tetraphosphate tetrahydrate. The piperaquine dose of $1280 \mathrm{mg}$ (piperaquine tetraphosphate equivalent) was given in the form of four $320 \mathrm{mg}$ strength tablets. The study consisted of a screening period of up to 26 days (Day -28 to -3 ), a baseline on Day -1 , followed by a single dose treatment in 3 parallel treatment arms on Day 1, and a study completion evaluation. The total duration for each subject to complete the study including baseline without screening was approximately 61 days.

Given the known food effect for piperaquine ( threefold increased exposure) and its QTc liability [20], all doses were given fasting. Subjects were admitted to the study site the night prior to dosing (approximately $12 \mathrm{~h}$ ) in each arm for baseline evaluations. Eligible subjects fasted (i.e., no food or liquid except for water) for at least $10 \mathrm{~h}$ prior to administration of study drug on Day 1 and continued to fast for at least $4 \mathrm{~h}$ thereafter. No fluid intake apart from the fluid given at the time of drug intake was allowed from $2 \mathrm{~h}$ before until $2 \mathrm{~h}$ after dosing. Lunch and dinner was served approximately 4 and 8 h post dosing, respectively.

Subjects were domiciled at the site from baseline until $48 \mathrm{~h}$ post-dose. Subjects then returned to the site at the follow-up visits detailed in the assessment schedule, for up to a 61-day period, to undergo safety evaluations and PK sampling. Study completion evaluation was conducted after the last PK sampling on Day 11 for KAF156 or Day 61 for arms containing piperaquine. 


\section{Subjects}

The study population comprised healthy males, aged 18-45 years of age and in good health as determined by past medical history, physical examination, vital signs, ECG, and laboratory tests at screening. Subjects weighed at least $50 \mathrm{~kg}$ to participate in the study, with a body mass index (BMI) within the range of $18-30 \mathrm{~kg} / \mathrm{m}^{2}$. The study was open to female subjects of non-childbearing potential, but none were recruited.

Exclusion criteria included use of other investigational drugs at the time of enrollment, or within five half-lives or within 30 days of enrollment; a history of clinically significant ECG abnormalities, screening/baseline Fridericia's formula corrected QT interval (QTcF) elevation (> $430 \mathrm{~ms}$ for males, > $440 \mathrm{~ms}$ for females); women of child-bearing potential; smokers or smokeless tobacco users who were unwilling or unable to refrain from tobacco use during confinement to the clinical research centre and during required study visits/evaluations; haemoglobin levels below $12.0 \mathrm{~g} / \mathrm{dL}$ at baseline; significant illness which did not resolve within 2 weeks prior to initial dosing; active disease; infections or conditions which may alter drug pharmacokinetics; renal or hepatic dysfunction; a history of drug or alcohol abuse within the 12 months prior to dosing, or clinical/laboratory evidence of such abuse.

A total of approximately $72(n=24 /$ arm $)$ subjects were planned to be enrolled and randomly assigned 1:1:1 into one of the three treatment arms:

- Arm I, 24 subjects received a single morning dose of $800 \mathrm{mg}$ KAF156 + a single dose of $1280 \mathrm{mg}$ piperaquine (KAF156 + PPQ).

- Arm II, 24 subjects received a single morning dose of 800 mg KAF156 (800 mg KAF156).

- Arm III, 24 subjects received a single morning dose of $1280 \mathrm{mg}$ piperaquine (1280 mg PPQ).

\section{Safety assessment}

Any potential relationship of drug exposure parameters to changes in ECG parameters was assessed. Safety assessments consisted of collecting all adverse events (AEs) and serious adverse events (SAEs), with their severity and relationship to study drug. They included regular monitoring of haematology, blood chemistry and urine performed at study centre and regular assessment of vital signs, physical condition, body weight and 12-lead electrocardiograms (ECG). Triplicate ECG assessments were performed at pre-dose and 2, 4, 8, 12,24 , and 48 h post-dose, while single ECGs were performed at the later time points (72 and $96 \mathrm{~h}$ and the end of study).

\section{Pharmacokinetic (PK) assessment}

Plasma concentrations were determined at pre-dose and then at $0.5,1,2,3,4,6,8,12,24,48,72,96,144,192$, and $240 \mathrm{~h}$ for both KAF156 and piperaquine. Additional samples were taken at 336, 504, 672, 1008 and $1440 \mathrm{~h}$ post dose for piperaquine. Venous blood samples were collected either through an indwelling catheter or by venipuncture into $\mathrm{K}_{2}$ EDTA-containing polyethylene tubes followed by gentle mixing and centrifugation between 2 and $8{ }^{\circ} \mathrm{C}$ for $10 \mathrm{~min}$ at approximately $1500 \mathrm{~g}$. Tubes were stored on wet ice or cryoblock until centrifuged (within $60 \mathrm{~min}$ ). Immediately after centrifugation, the supernatant plasma was transferred into $1.8 \mathrm{~mL}$ polypropylene screw-cap tubes which were placed in dry ice. The tubes were kept frozen at or below $-70{ }^{\circ} \mathrm{C}$ until bioanalysis. Plasma concentrations of KAF156 and piperaquine were determined by a validated liquid chromatographytandem mass spectrometry (LC-MS/MS) method; the Lower Limit of Quantification (LLOQ) is 5 and $0.5 \mathrm{ng} /$ $\mathrm{mL}$ for KAF156, and piperaquine, respectively.

The linearity ranges for KAF156 and PPQ are $1-5000 \mathrm{ng} / \mathrm{mL}$, and $0.5-250 \mathrm{ng} / \mathrm{mL}$, respectively. For the KAF156 assay, briefly, a $20.0 \mu \mathrm{L}$ aliquot plasma sample was mixed with a $25.0 \mu \mathrm{L}$ aliquot of the internal standard working solution [(M + 6)KAF156 $500 \mathrm{ng} / \mathrm{mL}$ in 50\% methanol]. A $200 \mu \mathrm{L}$ aliquot of acetonitrile (ACN) was added to the mixture. Subsequently, the sample was centrifuged at $2000 \mathrm{~g}$ for $10 \mathrm{~min}$ at $10{ }^{\circ} \mathrm{C}$. A $150 \mu \mathrm{L}$ aliquot of each supernatant was evaporated to dryness under nitrogen at $45{ }^{\circ} \mathrm{C}$. The sample was reconstituted in a $300 \mu \mathrm{L}$ aliquot of $\mathrm{MeOH}$-water-formic acid (FA) (10:89.9:0.1, $\mathrm{v} / \mathrm{v} / \mathrm{v})$. A $3.00 \mu \mathrm{L}$ aliquot of the sample was injected onto the LC-MS/MS system. The piperaquine method has already been described elsewhere [21].

The accuracy and precision for both KAF156 and piperaquine were within acceptable limits for study validation. For calibration standards, both KAF156 (5.00, 10.0, $50.0,250,500,1500,4000$ and $5000 \mathrm{ng} / \mathrm{mL}$ ) and piperaquine $(0.500,1.00,2.50,10.0,50.0,100,225$ and $250 \mathrm{ng} /$ $\mathrm{mL}$ ) had bias within the acceptable range of $\pm 20.0 \%$ at the LLOQ and $\pm 15.0 \%$ at the other concentration levels. Similarly, the 3 levels of quality control samples for both KAF156 and piperaquine had bias within the acceptable range of $\pm 15 \%$ for at least $2 / 3$ of the individual values.

The following PK parameters were determined using the actual recorded sampling times and non-compartmental method(s) with Phoenix WinNonlin (Version 6.4): Cmax, Tmax, AUClast, AUCinf, T1/2, Vz/F and $\mathrm{CL} / \mathrm{F}$ for KAF156 and piperaquine from the plasma concentration-time data.

The linear trapezoidal rule was used for AUC calculation. Regression analysis of the terminal plasma 
elimination phase for the determination of $\mathrm{T} 1 / 2$ included at least three data points after Cmax. If the adjusted $R^{2}$ value of the regression analysis of the terminal phase was to be less than 0.75 , no values were to be reported for T1/2, AUCinf and CL/F. If extrapolated AUCinf was more than $20 \%$ for KAF156 or more than $40 \%$ for piperaquine (due to its long terminal half-life) AUCinf and related parameters were not included in statistical analysis.

PK/PD relationships, using the individual concentrations and QTcF changes from baseline at each time point, were explored to establish the relationship between QTcF and drug exposure.

\section{Statistical methods}

Primary PK parameters were Cmax and AUClast. AUCinf was also estimated and reported; however, it was not considered as a primary PK parameter. The log-transformed primary PK parameters of KAF156 and piperaquine were analysed using a linear fixed effect model with treatment as the fixed effect. The analysis was performed using the natural $\log$ scale for PK parameters and the difference in adjusted means along with $90 \%$ confidence interval compared for the following:

- KAF156 $800 \mathrm{mg}$ with piperaquine $1280 \mathrm{mg}$ vs. KAF156 800 mg.

- KAF156 800 mg with piperaquine $1280 \mathrm{mg} v s$. piperaquine $1280 \mathrm{mg}$.

All results for the defined contrasts were back-transformed to the original scale to present adjusted geometric mean ratios and the corresponding $90 \%$ confidence intervals.

\section{Secondary variables}

\section{Analysis of ECG parameters over time points}

A key secondary endpoint for this trial was change from baseline in QTcF. Bazett's formula corrected QT interval (QTcB) analysis was limited to descriptive statistics and reported only as a summary table. The baseline was calculated from the pre-dose triplicate ECGs. At pre-dose and $2,4,8,12,24$, and $48 \mathrm{~h}$ post-dose triplicate ECG assessments were collected. The mean of triplicate QTcF values was calculated and used for all subsequent calculations and statistical evaluations. The endpoint for this analysis was calculated by subtracting the mean of triplicated QTcF pre-dose from post-dose assessment for each subject at each time point.

For 72 and $96 \mathrm{~h}$ post-dose time points, only a single assessment was performed. This endpoint was obtained by subtracting the mean of triplicate pre-dose QTcF value from the post-dose QTcF value for each subject and time point.

The change from baseline for QTcF for each time point was analysed using a linear model with treatment as the fixed effect and baseline (baseline was taken as $0 \mathrm{~h}$ on Day 1) as covariate in the model separately. The difference in adjusted means along with the $90 \%$ two-sided CI was calculated for KAF156 $800 \mathrm{mg}+$ piperaquine $1280 \mathrm{mg}$ vs. KAF156 $800 \mathrm{mg}$, and KAF156 $800 \mathrm{mg}$ + piperaquine $1280 \mathrm{mg}$ vs. piperaquine $1280 \mathrm{mg}$.

An arithmetic mean $( \pm$ standard deviation $(\mathrm{SD}))$ plot for change from baseline QT data (in Y axis) over several observed time points (in $\mathrm{X}$ axis) was performed for each treatment.

\section{Analysis of maximal change in ECG parameters}

A similar analysis was performed for maximal change from baseline for QTcF separately for KAF156 concentrations alone and in the presence of piperaquine, and for piperaquine concentrations alone and in the presence of KAF156, using a linear model with treatment as the fixed effect and concentration of KAF156 and piperaquine at maximal change as the covariate. The difference in adjusted means along with the $90 \%$ CI was calculated for KAF156 $800 \mathrm{mg}+$ piperaquine $1280 \mathrm{mg}$ vs. KAF156 $800 \mathrm{mg}$, and KAF156 $800 \mathrm{mg}+$ piperaquine $1280 \mathrm{mg}$ vs. piperaquine $1280 \mathrm{mg}$. For the endpoint maximal change from baseline the same model was explored using AUCinf or Cmax as covariate in the model instead of plasma concentration.

\section{Results}

\section{Subject demographics}

Seventy-two subjects were enrolled, 68 subjects (94.4\%) completed the study and 4 subjects (5.6\%) discontinued (2 subjects each in the KAF156 + PPQ arm and $1280 \mathrm{mg}$ PPQ arm). The primary reason for discontinuation was "subject/guardian decision". All subjects were male with a mean age of approximately 26 years old (range 19-45 years), and weight range 56.4-105.7 kg. $70.8 \%$ were Caucasian, $4.2 \%$ Black, 15.3\% Asian, and 9.7\% were other unspecified ethnicities. There were no major differences in demographic characteristics among treatment arms.

\section{Safety and tolerability}

There were no serious adverse events (SAEs) or deaths. The KAF156 + PPQ cohort had the highest incidence of adverse events $(21 / 24,87.5 \%)$, mostly attributable to nausea. This was followed by the PPQ cohort (19/24, $79.2 \%)$, then the KAF156 cohort (14/24, 58.3\%). In the KAF156 + PPQ cohort the most common AEs were 
nausea (41.7\%), upper respiratory tract infection (25.0\%), and headache (16.7\%). Headache was the most common AE in the KAF156 cohort (20.8\%) and PPQ cohorts (33.3\%) (Table 1).

Nausea was disproportionally higher in the KAF156 + PPQ arm (41.7\%) than the 800 mg KAF156
(12.5\%) or $1280 \mathrm{mg}$ PPQ (8.3\%) arms. Vomiting was present in a single subject in the KAF156 + PPQ and $1280 \mathrm{mg}$ PPQ arms only (4.2\%), with vomiting in the KAF156 + PPQ arm occurring minutes after dosing. Most subjects with AEs had Grade 1 events. A total of 16 subjects had at least one Grade $2 \mathrm{AE}$. There were no Grade 3 or 4 events.

Table 1 Incidence of adverse events by preferred term

\begin{tabular}{|c|c|c|c|c|}
\hline Preferred term & $\begin{array}{l}800 \mathrm{mg} \text { KAF156 + } 1280 \mathrm{mg} \text { PPQ } \\
N=24 \\
n(\%)\end{array}$ & $\begin{array}{l}800 \mathrm{mg} \mathrm{KAF} 156 \\
N=24 \\
n(\%)\end{array}$ & $\begin{array}{l}1280 \mathrm{mg} P P Q \\
\mathrm{~N}=24 \\
\mathrm{n}(\%)\end{array}$ & $\begin{array}{l}\text { Total } \\
\mathrm{N}=72 \\
\mathrm{n}(\%)\end{array}$ \\
\hline Number of subjects with at least one AE & $21(87.5)$ & $14(58.3)$ & $19(79.2)$ & $54(75.0)$ \\
\hline Headache & $4(16.7)$ & $5(20.8)$ & $8(33.3)$ & $17(23.6)$ \\
\hline Nausea & $10(41.7)$ & $3(12.5)$ & $2(8.3)$ & $15(20.8)$ \\
\hline Upper respiratory tract infection & $6(25.0)$ & $2(8.3)$ & $7(29.2)$ & $15(20.8)$ \\
\hline Abdominal pain & $3(12.5)$ & $1(4.2)$ & 0 & $4(5.6)$ \\
\hline Dizziness & $2(8.3)$ & 0 & $2(8.3)$ & $4(5.6)$ \\
\hline Diarrhoea & $1(4.2)$ & $1(4.2)$ & $1(4.2)$ & $3(4.2)$ \\
\hline Dyspepsia & $1(4.2)$ & $1(4.2)$ & $1(4.2)$ & $3(4.2)$ \\
\hline Somnolence & 0 & $1(4.2)$ & $2(8.3)$ & $3(4.2)$ \\
\hline Fatigue & $1(4.2)$ & 0 & $1(4.2)$ & $2(2.8)$ \\
\hline Pyrexia & 0 & 0 & $2(8.3)$ & $2(2.8)$ \\
\hline Visual impairment & $1(4.2)$ & $1(4.2)$ & 0 & $2(2.8)$ \\
\hline Vomiting & $1(4.2)$ & 0 & $1(4.2)$ & $2(2.8)$ \\
\hline Abdominal discomfort & $1(4.2)$ & 0 & 0 & $1(1.4)$ \\
\hline Alanine aminotransferase increased & 0 & $1(4.2)$ & 0 & $1(1.4)$ \\
\hline Catheter site erythema & 0 & $1(4.2)$ & 0 & $1(1.4)$ \\
\hline Conjunctival haemorrhage & 0 & $1(4.2)$ & 0 & $1(1.4)$ \\
\hline Cough & 0 & $1(4.2)$ & 0 & $1(1.4)$ \\
\hline Dry skin & 0 & 0 & $1(4.2)$ & $1(1.4)$ \\
\hline Dyskinesia & 0 & 0 & $1(4.2)$ & $1(1.4)$ \\
\hline Foreign body & $1(4.2)$ & 0 & 0 & $1(1.4)$ \\
\hline Gastroenteritis & 0 & $1(4.2)$ & 0 & $1(1.4)$ \\
\hline Gastroesophageal reflux disease ${ }^{a}$ & 0 & 0 & $1(4.2)$ & $1(1.4)$ \\
\hline Infected bite & $1(4.2)$ & 0 & 0 & $1(1.4)$ \\
\hline Insomnia & 0 & 0 & $1(4.2)$ & $1(1.4)$ \\
\hline Ligament sprain & $1(4.2)$ & 0 & 0 & $1(1.4)$ \\
\hline Lymphadenopathy & $1(4.2)$ & 0 & 0 & $1(1.4)$ \\
\hline Muscle spasms & 0 & 0 & $1(4.2)$ & $1(1.4)$ \\
\hline Myalgia & 0 & 0 & $1(4.2)$ & $1(1.4)$ \\
\hline Neck pain & $1(4.2)$ & 0 & 0 & $1(1.4)$ \\
\hline Oropharyngeal pain & 0 & 0 & $1(4.2)$ & $1(1.4)$ \\
\hline Phlebitis & 0 & $1(4.2)$ & 0 & $1(1.4)$ \\
\hline Rash erythematous & $1(4.2)$ & 0 & 0 & $1(1.4)$ \\
\hline Sleep disorder & 0 & 0 & $1(4.2)$ & $1(1.4)$ \\
\hline Toothache & 0 & 0 & $1(4.2)$ & $1(1.4)$ \\
\hline Viral infection & 0 & 0 & $1(4.2)$ & $1(1.4)$ \\
\hline
\end{tabular}

A subject with multiple adverse events (AEs) is counted only once in the "at least one AE" row

A subject with multiple AEs with the same preferred term is counted only once for that preferred term \& treatment

Preferred terms are sorted in descending frequency

a Reflux oesophagitis began before dosing but was exacerbated following dosing and labelled 'drug-related' 
Laboratory abnormalities were generally not of clinical significance. Hypereosinophilia up to $2.33 \times$ ULN was seen distributed across all dosing arms in up to 23 subjects with no apparent difference among arms. This study was conducted during hay fever season in Melbourne and none of the cases were considered clinically-significant. Elevated creatine kinase levels (ranging from Grade 1 to Grade 3 abnormalities), occurred in most of the affected subjects before study drug administration, was not associated with AEs, was generally transitory, and occurred across dosing arms with no obvious pattern or dose relationship. There was an AE of Grade 1 elevated AST, suspected to be drug-related occurring in one subject in the $800 \mathrm{mg}$ KAF156 arm. All his ALT readings, including those at screening and baseline were also Grade 1 elevations. Vital signs showed no significant changes in mean/ median values over time which were within normal ranges, and no notable differences among the treatment arms.

\section{ECG results}

\section{Individual results}

There were no ECG related AEs, no QTcF interval $>480 \mathrm{~ms}$, and no QTcF interval increase from baseline $>60 \mathrm{~ms}$. There was no difference in heart rate among the treatment arms. Mean maximal change from baseline (Table 2): Maximum increase in QTcF from baseline was observed at $24 \mathrm{~h}$ post-dose for both KAF156 + PPQ and KAF156 monotherapy arm; the mean increases from pre-dose (Day $1-0 \mathrm{~h}$ ) was 7.9 and $6.6 \mathrm{~ms}$, respectively. For the PPQ monotherapy arm, the maximum increase from pre-dose was found to be $1.8 \mathrm{~ms}$ (mean) at $4 \mathrm{~h}$ postdose. The maximum QTcF change, irrespective of time point, was highest in the KAF156 + PPQ arm (median $13.7 \mathrm{~ms}$, maximum $26 \mathrm{~ms}$ ) followed by the $800 \mathrm{mg}$ KAF156 arm (median $8.0 \mathrm{~ms}$, maximum $44 \mathrm{~ms}$ ) then the $1280 \mathrm{mg}$ PPQ (median $7.0 \mathrm{~ms}$, maximum $23.7 \mathrm{~ms}$ ). The KAF156 + PPQ arm also had the highest mean change (12.5 ms) compared with the single treatment arms (800 mg KAF156:10.8 ms; 1280 mg PPQ:8.2 ms).

Concentration effect relationship for QTcF: To understand the concentration effect relationship, individual concentrations vs. $\triangle \mathrm{QTCF}$ on specific time points and maximum QTcF, change from pre-dose $(\triangle \mathrm{QTcF})$ vs. Cmax were plotted. Additionally, an ANCOVA model was fitted over pre-dose corrected QTcF values, with Cmax (of KAF156 then piperaquine) as covariate and respective treatments as fixed effects. There was a positive $\Delta$ QTcF trend in the KAF156 + PPQ arm when either KAF156 or piperaquine concentration increases, but there was no significant difference between the combination arm and other arms in maximum $\Delta \mathrm{QTcF}(\mathrm{p}>0.05)$.
Table 2 Summary statistics of maximal change from predose in QTcF (safety analysis set)

\begin{tabular}{lll}
\hline Treatment & Statistics & $\begin{array}{l}\text { QTcF change from pre- } \\
\text { dose (msec) }\end{array}$ \\
\hline $\begin{array}{ll}\text { KAF156 } 800 \mathrm{mg}+\mathrm{PPQ} \\
1280 \mathrm{mg}\end{array}$ & $\mathrm{N}$ & 24 \\
& Mean (SD) & $12.5(7.84)$ \\
& CV\% & 62.51 \\
& Median & 13.7 \\
KAF156 $800 \mathrm{mg}$ & (Min, max) & $(-8.7,26.00)$ \\
& N & 24 \\
& Mean (SD) & $10.8(9.83)$ \\
& CV\% & 91.18 \\
Median & 8.0 \\
(Min, max) & $(-1.7,44.00)$ \\
NPQ $1280 \mathrm{mg}$ & 24 \\
& Mean (SD) & $8.2(8.29)$ \\
& CV\% & 101.63 \\
Median & 7.0 \\
(Min, max) & $(-7.3,23.67)$ \\
\hline
\end{tabular}

$\mathrm{QTcF}=$ Maximum change from post treatment in QTcF

In Fig. 1 the potential concentration-effect relationships for QTcF change from baseline is explored as individual KAF156 (panel A) or piperaquine (panel B) concentrations vs. maximal change from baseline in QTcF. In both analyses the slope of the relationship is not significantly different for the monotherapy vs. combination comparison. Therefore, no evidence of a synergistic effect on QTc interval when KAF156 and piperaquine are combined was found.

\section{Pharmacokinetic assessment}

Figures 2, 3 show the exposure vs. time profiles for KAF156 and piperaquine when administered as either a monotherapy or part of the combination therapy. The corresponding PK parameters are shown in Tables 3 and 4 . There was no noteworthy alteration in the extent of exposure (AUC) of KAF156 and piperaquine when given in combination compared to their administration as single agent. KAF156 Cmax was 1.23-fold (90\% CI 1.10, 1.37) higher in the combination arm compared to KAF156 alone arm. Piperaquine Cmax was 1.69 (90\% CI 1.16, 2.45) fold higher in the combination arm compared to monotherapy arm however it should be noted that piperaquine $\mathrm{Cmax}$ was highly variable (CV\% 70\%). There was no increase in the T1/2 of either drug when given in combination compared to their single drug arms; however, there was shortening of either median Tmax or range of Tmax for both the drugs in combination compared to their respective single drug arms. 


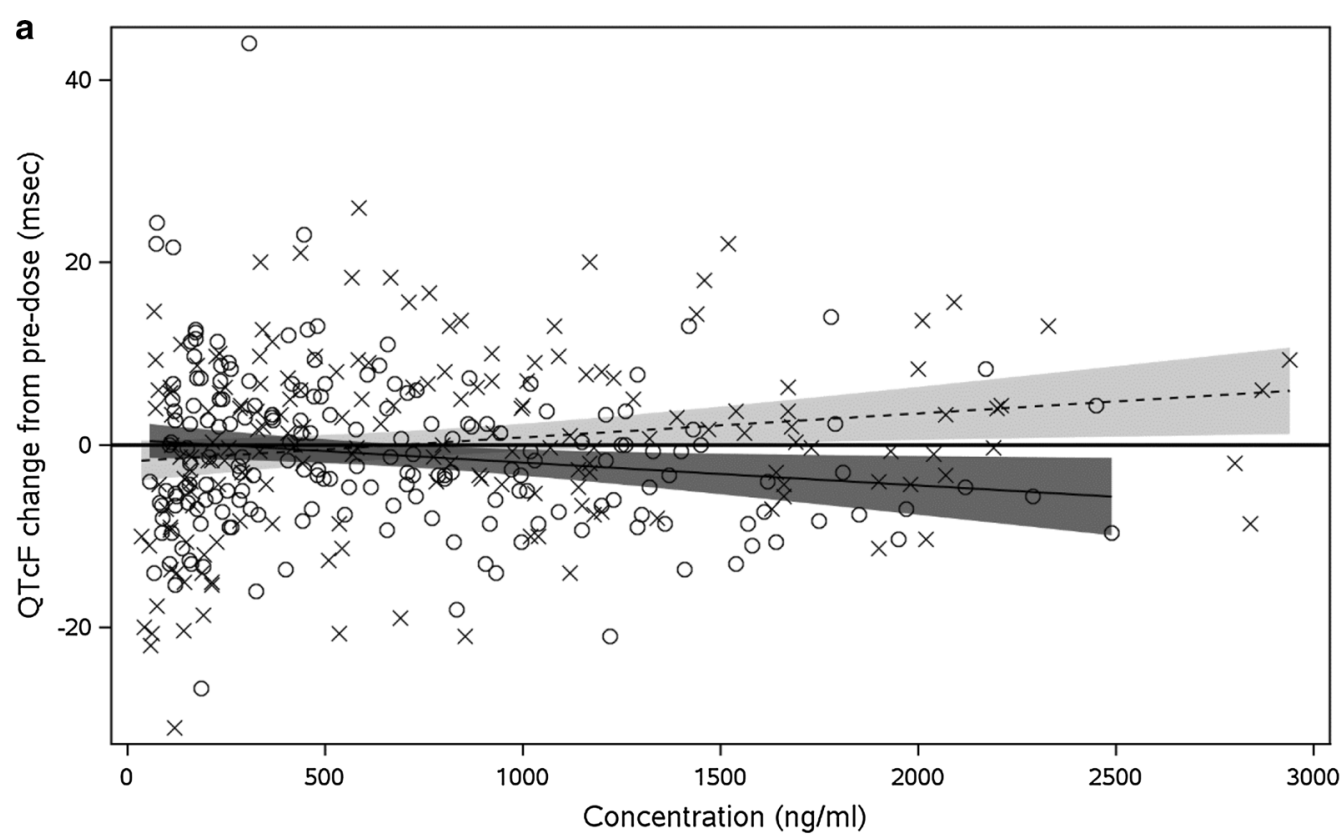

\begin{tabular}{|clcl|}
\hline$\times$ & Individual Conc (Treatment: KAF156 + Piperaquine) & ---- & Treatment: KAF156 + Piperaquine \\
$\square$ & $95 \%$ Confidence Limits & $\circ$ & Individual Conc (Treatment: KAF156) \\
\hline & Treatment: KAF156 & $\square$ & $95 \%$ Confidence Limits \\
\hline
\end{tabular}

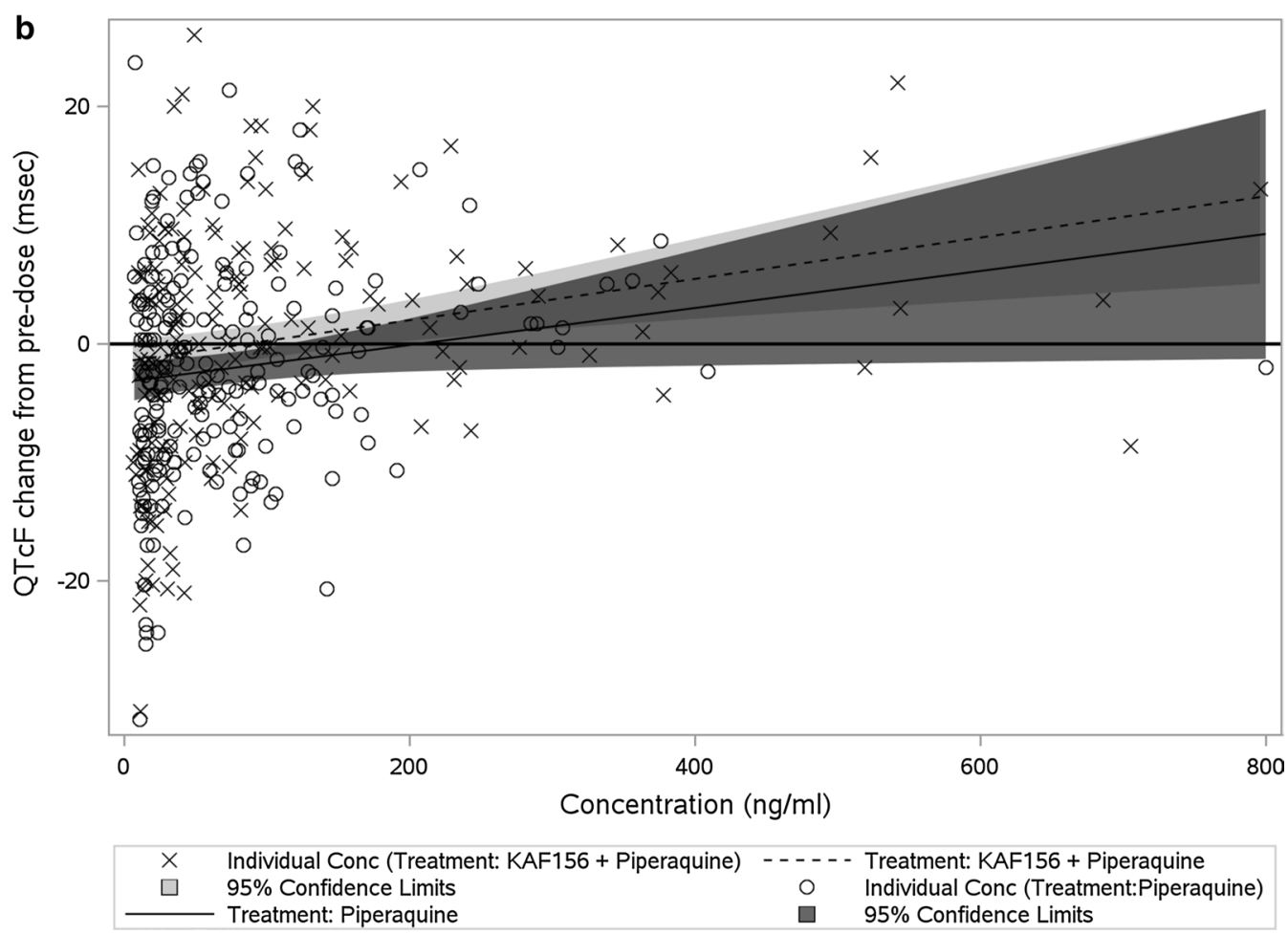

Fig. 1 Individual drug concentration and its relationship with QTCF change from baseline when given as monotherapy and as combination; a for KAF156, b for PPQ. Open circles represent the monotherapy and cross marks represent the combination treatment. The dark gray and light gray shaded areas represent the $95 \%$ confidence interval for monotherapy and combination respectively 


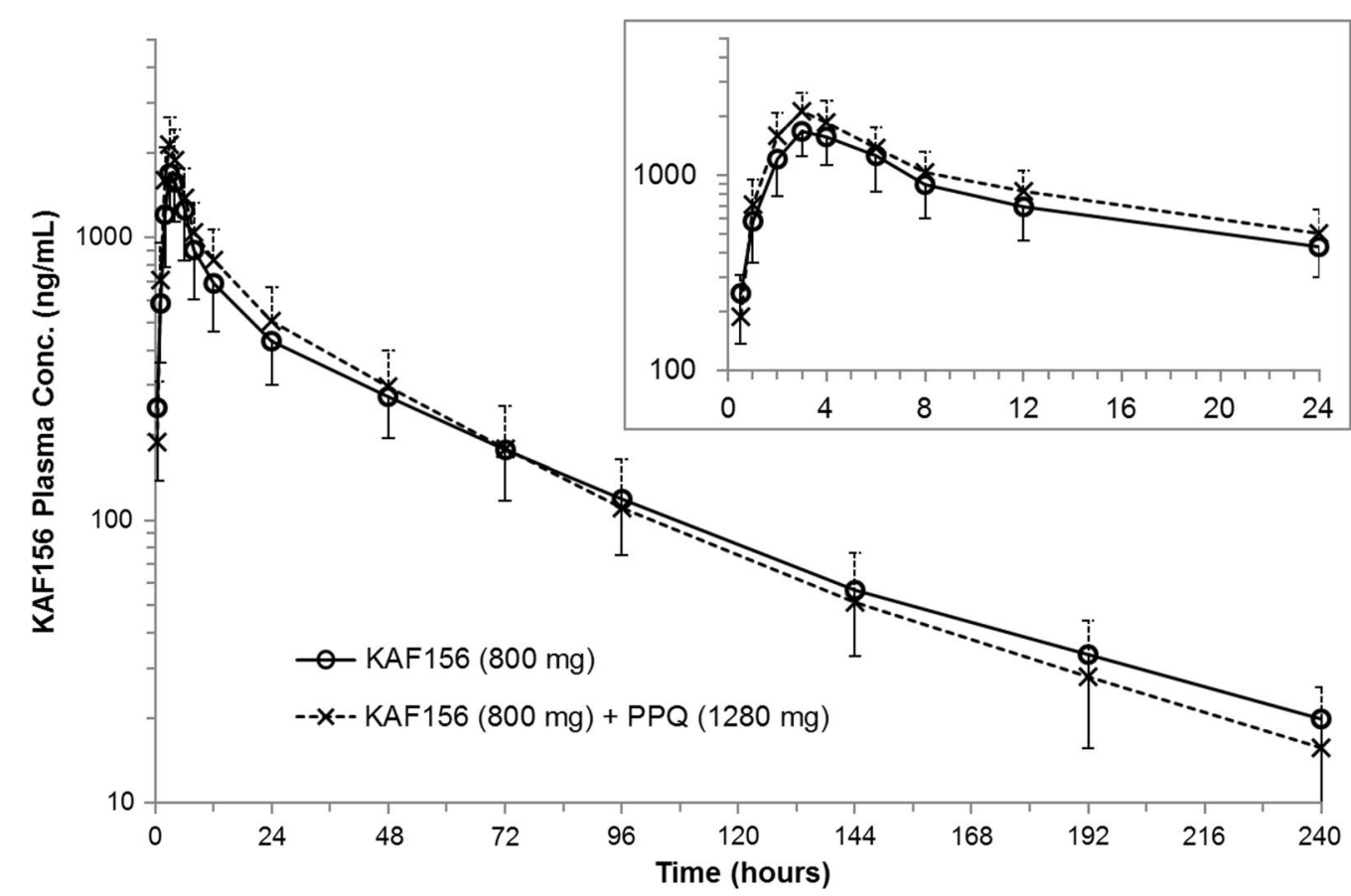

Fig. 2 Arithmetic mean (SD) plasma concentration time profiles for KAF156 according to time and treatment group. Inset, first $24 \mathrm{~h}$ after dosing. Solid lines with open circles represent the KAF156 concentrations when given as monotherapy and dashed lines with cross marks represent KAF156 concentrations when given in combination with PPQ

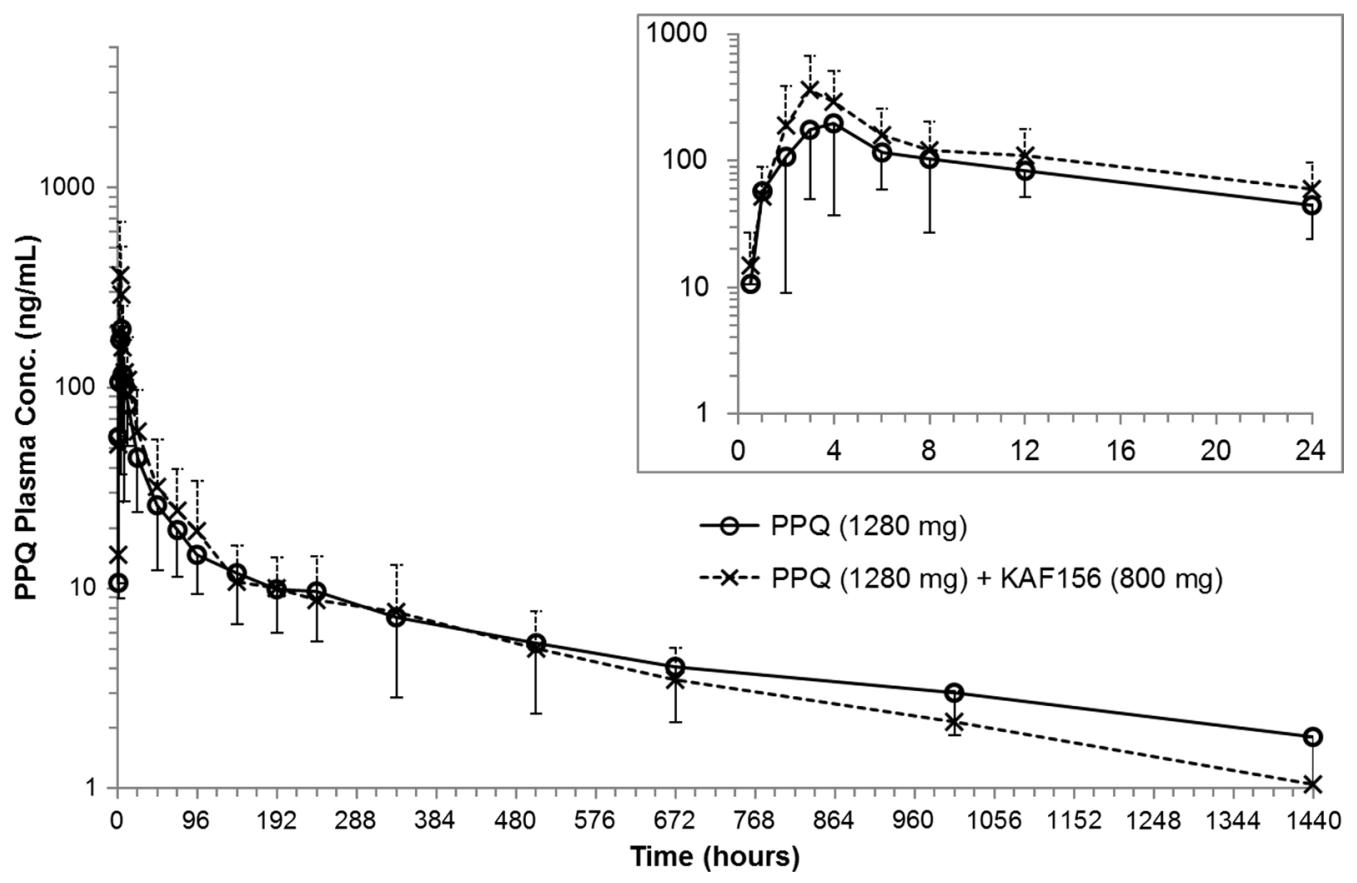

Fig. 3 Arithmetic mean (SD) plasma concentration time profiles for piperaquine according to time and treatment group. Inset, first $24 \mathrm{~h}$ after dosing. Solid lines with open circles represent the PPQ concentrations when given as monotherapy and dashed lines with cross marks represent PPQ concentrations when given in combination with KAF156 
Table 3 PK parameters for KAF156 in the presence and absence of piperaquine

\begin{tabular}{|c|c|c|}
\hline PK parameter ${ }^{\mathrm{a}}$ (unit) & $\begin{array}{l}800 \text { mg KAF156 + } 1280 \mathrm{mg} \text { PPQ } \\
\mathrm{N}=23 \text { (mean, SD, CV\%) }\end{array}$ & $\begin{array}{l}800 \text { mg KAF156 } \\
\mathrm{N}=24 \text { (mean, SD, CV\%) }\end{array}$ \\
\hline $\mathrm{Cmax}(\mathrm{ng} / \mathrm{mL})$ & $2270 \pm 496(21.8 \%)[n=23]$ & $1850 \pm 401(21.7 \%)[n=24]$ \\
\hline AUClast (h $\mu \mathrm{g} / \mathrm{mL})$ & $48.2 \pm 14.4(30.0 \%)[n=22]$ & $44.4 \pm 12.7(28.7 \%)[n=24]$ \\
\hline AUCinf $(h \mu g / m L)$ & $50.1 \pm 15.0(29.9 \%)[n=21]$ & $46.2 \pm 13.9(30.0 \%)[n=24]$ \\
\hline AUC0-24 h (h $\mu \mathrm{g} / \mathrm{mL})$ & $22.8 \pm 5.41(23.7 \%)[n=22]$ & $19.1 \pm 4.86(25.4 \%)[n=24]$ \\
\hline AUC0-72 h (h $\mu \mathrm{g} / \mathrm{mL})$ & $38.2 \pm 10.2(26.6 \%)[n=22]$ & $33.0 \pm 8.67(26.2 \%)[n=24]$ \\
\hline AUC0-168 h (h $\mu \mathrm{g} / \mathrm{mL})$ & $47.0 \pm 13.7(29.0 \%)[n=21]$ & $42.2 \pm 11.8(27.9 \%)[n=24]$ \\
\hline $\operatorname{Tmax}(\mathrm{h})$ & $3.00(2.00-4.02)[n=23]$ & $3.00(2.00-6.00)[n=24]$ \\
\hline $\mathrm{T} 1 / 2(h)$ & $52.4 \pm 11.1(21.2 \%)[n=21]$ & $57.9 \pm 10.5(18.2 \%)[n=24]$ \\
\hline$C L / F(m L / h)$ & $17,500 \pm 5760(32.9 \%)[n=21]$ & $18,700 \pm 5210(27.8 \%)[n=24]$ \\
\hline $\mathrm{V} z / \mathrm{F}(\mathrm{L})$ & $1360 \pm 689(50.7 \%)[n=21]$ & $1530 \pm 417(27.2 \%)[n=24]$ \\
\hline
\end{tabular}

AUCO- $\mathrm{t} h$, The area under the plasma concentration-time curve from time zero to time ' $\mathrm{t}$ ' where $\mathrm{t}$ is a defined time point after administration

AUCinf, The area under the plasma concentration-time curve from time zero to infinity

AUClast, The area under the plasma concentration-time curve from time zero to the time of the last quantifiable concentration

Cmax, The observed maximum plasma concentration following drug administration

$\mathrm{CL} / \mathrm{F}$, The apparent systemic (or total body) clearance from plasma following extravascular administration

$\mathrm{T} 1 / 2$, The terminal elimination half-life

Tmax, The time to reach the maximum concentration after drug administration

$\mathrm{V} z / F$, The apparent volume of distribution during the terminal elimination phase following extravascular administration

${ }^{a}$ All PK parameter values are presented as mean \pm SD (CV \%) [n] except Tmax which is presented as median (range) [n]. $n$ is number of subjects providing reliable estimate of the parameter

Table 4 PK parameters for piperaquine in the presence and absence of KAF156

\begin{tabular}{|c|c|c|}
\hline PK parameter ${ }^{\mathrm{a}}$ (unit) & $\begin{array}{l}800 \text { mg KAF156 + } 1280 \text { mg PPQ } \\
N=23 \text { (mean, SD, CV\%) }\end{array}$ & $\begin{array}{l}1280 \mathrm{mg} \text { PPQ } \\
\mathrm{N}=24 \text { (mean, SD, CV\%) }\end{array}$ \\
\hline Cmax (ng/mL) & $409 \pm 299(73.2 \%)[n=23]$ & $233 \pm 169(72.3 \%)[n=24]$ \\
\hline AUClast (h $\mu \mathrm{g} / \mathrm{mL})$ & $10.8 \pm 6.14(56.8 \%)[n=22]$ & $10.3 \pm 4.16(40.2 \%)[n=23]$ \\
\hline AUCinf $(h \mu g / m L)$ & $11.9 \pm 5.25(44.1 \%)[n=19]$ & $12.0 \pm 5.31(44.2 \%)[n=17]$ \\
\hline AUC0-24 h (h $\mu \mathrm{g} / \mathrm{mL})$ & $2.94 \pm 1.81(61.5 \%)[n=22]$ & $2.09 \pm 0.993(47.4 \%)[n=24]$ \\
\hline AUC0-72 h (h $\mu \mathrm{g} / \mathrm{mL})$ & $4.73 \pm 2.89(61.0 \%)[n=22]$ & $3.49 \pm 1.56(44.8 \%)[n=24]$ \\
\hline AUCO-168 h (h $\mu \mathrm{g} / \mathrm{mL})$ & $6.35 \pm 3.67(57.9 \%)[n=21]$ & $4.82 \pm 1.95(40.4 \%)[n=23]$ \\
\hline $\operatorname{Tmax}(h)$ & $3.00(2.00-4.05)[n=23]$ & $4.00(3.00-8.00)[n=24]$ \\
\hline $\mathrm{T} 1 / 2(\mathrm{~h})$ & $469 \pm 170(36.2 \%)[n=19]$ & $509 \pm 142(27.9 \%)[n=17]$ \\
\hline $\mathrm{CL} / \mathrm{F}(\mathrm{mL} / \mathrm{h})$ & $127,000 \pm 53,100(41.7 \%)[n=19]$ & $126,000 \pm 52,700(41.9 \%)[n=17]$ \\
\hline $\mathrm{V} z / \mathrm{F}(\mathrm{L})$ & $82,200 \pm 34,500(42.0 \%)[n=19]$ & $89,100 \pm 32,800(36.8 \%)[n=17]$ \\
\hline
\end{tabular}

Abbreviations as per Table 3

a All PK parameter values are presented as mean \pm SD (CV\%) [n] except Tmax which is presented as median (range) [n]. $n$ is number of subjects providing reliable estimate of the parameter

\section{Discussion}

This study examined the safety, potential pharmacokinetic and QTcF interactions of KAF156 and piperaquine when dosed alone or in combination. There were no clinically relevant AE's found for either agent when dosed either alone or in combination (Table 1) and the safety profiles resembled past studies performed with KAF156 $[12,13]$ or piperaquine given as a single dose in healthy volunteers [21].
One of the major safety concerns for the use of these agents in combination is the potential for QT prolongation given the known risk for piperaquine and the potential risk from in vitro screens of KAF156 (e.g., hERG inhibition). For this reason, the study incorporated triplicate ECG analyses and planned to evaluate ECG changes as a function of exposure response relationships. There was no evidence of a synergistic effect when the agents were given together: piperaquine 


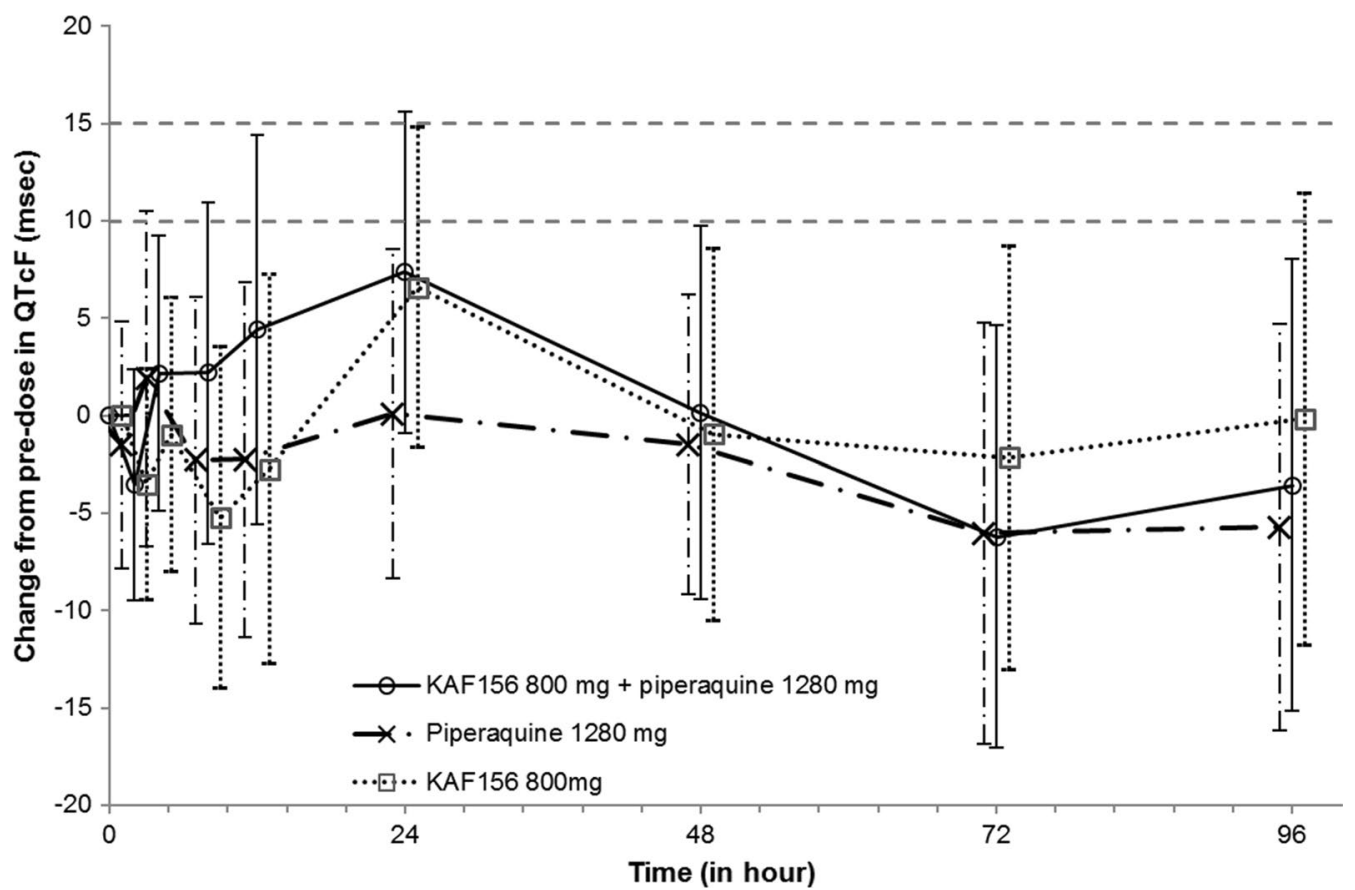

Fig. 4 Arithmetic mean (SD) QTcF change time profile from pre-dose profile by treatment. Combination is indicated by the solid line with circles, KAF156 monotherapy by the dotted line with squares, and piperaquine monotherapy by the dash-dot line with cross marks

but not KAF156 had a positive slope for exposure vs. increase in QTcF.

In a previous study examining the safety, PK, and PD relationship for KAE609 and piperaquine [21], the mean maximal change from baseline for piperaquine in the regression model was $7.47 \mathrm{~ms}$, which is similar to the $8.49 \mathrm{~ms}$ observed in this trial. The overall change from baseline vs time for QTcF (Fig. 4) for piperaquine is less consistent than in the earlier study and the difference between the piperaquine and combination arms was greater in that study. It is unclear why these differences between studies were found as the study site, use of triplicate ECGs and analysis are the same. The main difference is that given the longer half-life of KAF156 compared with KAE609, triplicate ECGs were performed for a longer time interval after dosing in this trial.

The PK profiles of each agent are consistent with the prior data for each agent $[12,21]$ when dosed under fasting conditions in healthy volunteers. There were statistically significant increases in the Cmax values of KAF156, 1.23-fold (90\% CI 1.10, 1.37), and piperaquine, 1.69 -fold ( $90 \%$ CI 1.16, 2.45), when dosed in combination. Given the lack of a relationship of the increased Cmax values of each agent to QTcF increase, as noted in the earlier analysis, it is unlikely that there is clinical relevance to the Cmax increase observed for either drug.
Overall, no safety/cardiac risk or drug-drug interaction was identified which would preclude use of a KAF156 and PPQ combination in future studies. As both KAF156 and piperaquine have demonstrated efficacy in prior clinical trials, the combination could be explored in clinical trials without dose adjustment or concern for increased cardiac arrhythmia risk as a non-cross resistant alternative to artemisinin combination therapy in the treatment of blood-stage falciparum malaria.

\section{Authors' contributions}

FJL wrote the first draft of this manuscript. All authors were part of the Novartis clinical team which wrote the study. All authors read and approved the final manuscript.

\section{Author details}

${ }^{1}$ Novartis Institute for Tropical Diseases, Singapore, Singapore. ${ }^{2}$ Novartis Healthcare Pvt Ltd, Hyderabad, India. ${ }^{3}$ Novartis Institutes for BioMedical Research, Shanghai, People's Republic of China. ${ }^{4}$ Novartis Pharma, East Hanover, NJ, USA. ${ }^{5}$ Present Address: D3, Agency for Science, Technology and Research (A*STAR), Singapore, Singapore.

\section{Acknowledgements}

The study was sponsored by Novartis. We would like to acknowledge the work of Dr. Jason Lickliter, and the clinical team at Nucleus Network Limited, Melbourne, Australia.

\section{Competing interests}

All authors are/were Novartis employees during the time of the study and the manuscript writing. 


\section{Availability of data and materials}

All key data generated or analysed during this study are included in this published article.

\section{Consent for publication}

Not applicable.

\section{Ethics approval and consent to participate}

This study was conducted in compliance with the Declaration of Helsinki and the International Conference on Harmonization Guidelines for Good Clinical Practice. The final protocol, amendments, and informed consent documentation were reviewed and approved by the Belberry Human Research Ethics Committee (129 Glen Osmond Rd Eastwood, South Australia). All subjects provided written, informed consent before participating in any study procedures.

\section{Funding}

This study was sponsored by Novartis.

\section{Publisher's Note}

Springer Nature remains neutral with regard to jurisdictional claims in published maps and institutional affiliations.

Received: 23 June 2017 Accepted: 23 December 2017

Published online: 05 January 2018

\section{References}

1. WHO. World Malaria Report 2016. Geneva: World Health Organization; 2016. p. 186. http://www.who.int/malaria/publications/world-malariareport-2016/report/en/. Accessed 2 Jan 2018

2. Ashley EA, Dhorda M, Fairhurst RM, Amaratunga C, Lim P, Suon S, et al. Spread of artemisinin resistance in Plasmodium falciparum malaria. N Engl J Med. 2014;371:411-23.

3. White NJ. Malaria: a molecular marker of artemisinin resistance. Lancet. 2014;383:1439-40.

4. Menard D, Ariey F. Towards real-time monitoring of artemisinin resistance. Lancet Infect Dis. 2015;15:367-8.

5. Dondorp AM, Nosten F, Yi P, Das D, Phyo AP, Tarning J, et al. Artemisinin resistance in Plasmodium falciparum malaria. N Engl J Med. 2009;361:455-67.

6. Noedl $\mathrm{H}$. The need for new antimalarial drugs less prone to resistance. Curr Pharm Des. 2013;19:266-9.

7. Tun KM, Imwong M, Lwin KM, Win AA, Hlaing TM, Hlaing T, et al Spread of artemisinin-resistant Plasmodium falciparum in Myanmar: a cross-sectional survey of the K13 molecular marker. Lancet Infect Dis. 2015;15:415-21.

8. Packard RM. The origins of antimalarial-drug resistance. N Engl J Med. 2014;371:397-9.
9. Nagle A, Wu T, Kuhen K, Gagaring K, Borboa R, Francek C, et al. Imidazolopiperazines: lead optimization of the second-generation antimalarial agents. J Med Chem. 2012;55:4244-73.

10. Wu T, Nagle A, Kuhen K, Gagaring K, Borboa R, Francek C, et al. Imidazolopiperazines: hit to lead optimization of new antimalarial agents. J Med Chem. 2011;54:5116-30.

11. Kuhen KL, Chatterjee AK, Rottmann M, Gagaring K, Borboa R, Buenviaje J, et al. KAF156 is an antimalarial clinical candidate with potential for use in prophylaxis, treatment, and prevention of disease transmission. Antimicrob Agents Chemother. 2014;58:5060-7.

12. Leong FJ, Zhao R, Zeng S, Magnusson B, Diagana TT, Pertel P. A firstin-human randomized, double-blind, placebo-controlled, single- and multiple-ascending oral dose study of novel Imidazolopiperazine KAF156 to assess its safety, tolerability, and pharmacokinetics in healthy adult volunteers. Antimicrob Agents Chemother. 2014;58:6437-43.

13. White NJ, Duong TT, Uthaisin C, Nosten F, Phyo AP, Hanboonkunupakarn $\mathrm{B}$, et al. Antimalarial activity of KAF156 in falciparum and vivax malaria. N Engl J Med. 2016:375:1152-60.

14. Plucinski MM, Talundzic E, Morton L, Dimbu PR, Macaia AP, Fortes F, et al. Efficacy of artemether-lumefantrine and dihydroartemisinin-piperaquine for treatment of uncomplicated malaria in children in Zaire and Uíge provinces, angola. Antimicrob Agents Chemother. 2015:59:437-43.

15. Myint HY, Ashley EA, Day NPJ, Nosten F, White NJ. Efficacy and safety of dihydroartemisinin-piperaquine. Trans R Soc Trop Med Hyg. 2007;101:858-66

16. Keating GM. Dihydroartemisinin/Piperaquine: a review of its use in the treatment of uncomplicated Plasmodium falciparum malaria. Drugs. 2012;72:937-61.

17. Reuter SE, Evans AM, Shakib S, Lungershausen Y, Francis B, Valentini G, et al. Effect of food on the pharmacokinetics of piperaquine and dihydroartemisinin. Clin Drug Investig. 2015;35:559-67.

18. European Medicines Agency. Eurartesim. EPAR summary for the public. European Medicines Agency; 2016 Jul. Report No.: EMA/526898/2016 EMEA/H/C/001199. http://www.ema.europa.eu/ema/index. jsp?curl=pages/medicines/human/medicines/001199/human_ med_001450.jsp\&mid=WC0b01ac058001d124. Accessed 2 Jan 2018

19. Baiden R, Oduro A, Halidou T, Gyapong M, Sie A, Macete E, et al. Prospective observational study to evaluate the clinical safety of the fixed-dose artemisinin-based combination Eurartesim ${ }^{\circledR}$ (dihydroartemisinin/piperaquine), in public health facilities in Burkina Faso, Mozambique, Ghana, and Tanzania. Malar J. 2015;14:160.

20. European Medicines Agency_Eurartesim—Summary of product characteristics. http://www.ema.europa.eu/docs/en_GB/document_library/ EPAR_-_Product_Information/human/001199/WC500118113.pdf. Accessed 15 May 2017

21. Stein DS, Jain JP, Kangas M, Lefèvre G, Machineni S, Griffin P, et al. Openlabel, single-dose, parallel-group study in healthy volunteers to determine the drug-drug interaction potential between KAE609 (cipargamin) and piperaquine. Antimicrob Agents Chemother. 2015;59:3493-500.

\section{Submit your next manuscript to BioMed Central and we will help you at every step:}

- We accept pre-submission inquiries

- Our selector tool helps you to find the most relevant journal

- We provide round the clock customer support

- Convenient online submission

- Thorough peer review

- Inclusion in PubMed and all major indexing services

- Maximum visibility for your research

Submit your manuscript at www.biomedcentral.com/submit 